\title{
Pacific
}

Journal of

Mathematics

\section{ISOMORPHISMS OF SPACES OF CONTINUOUS AFFINE FUNCTIONS}

\author{
Cho-Ho Chu And Henry Bruce Cohen
}

Volume $155 \quad$ No. 1 


\title{
ISOMORPHISMS OF SPACES OF CONTINUOUS AFFINE FUNCTIONS
}

\author{
Cho-Ho Chu and Henry B. Cohen
}

Let $K$ and $S$ be compact convex sets and let $A(K)$ and $A(S)$ be the corresponding Banach spaces of continuous affine functions. If the Banach-Mazur distance between $A(K)$ and $A(S)$ is less than 2 , then under certain geometric conditions, the extreme boundaries of $K$ and $S$ are homeomorphic. This extends a result of Amir and Cambern, and has applications to function algebras.

1. Introduction. Let $X$ and $Y$ be compact Hausdorff spaces. The classical Banach-Stone theorem states that if the spaces $C(X)$ and $C(Y)$ of (real) continuous functions are isometric, then $X$ and $Y$ are homeomorphic. There have been several generalizations of this theorem, among which is the following result of Amir [2] and Cambern [9]:

If there is a (surjective linear) isomorphism $\varphi: C(X) \rightarrow C(Y)$ such that $\|\varphi\|\left\|\varphi^{-1}\right\|<2$, then $X$ and $Y$ are homeomorphic.

Alternative proofs of this result have been given by Cohen [12] and Drewnowski [13]. The result is false if the bound $\|\varphi\|\left\|\varphi^{-1}\right\|$ is not less than 2 [11] although it has been generalized to spaces of vectorvalued continuous functions (cf. $[5,7,10,15,17])$. Nevertheless, in an attempt to extend the result to certain function spaces, for instance, to function algebras, we found that the arguments used in [9] and [12] can actually be adapted to a setting more general than function algebras which not only yields more general results but also gives new insight into the essentials of these arguments.

Our setting is that of compact convex sets $K$ (in locally convex spaces) and the Banach spaces $A(K)$ of (real) continuous affine functions on $K$. We will show, given two compact convex sets $K$ and $S$ with extreme boundaries $\partial K$ and $\partial S$ respectively, if there is an isomorphism $\varphi: A(K) \rightarrow A(S)$ satisfying $\|\varphi\|\left\|\varphi^{-1}\right\|<2$, then any one of the following conditions implies that $\partial K$ and $\partial S$ are homeomorphic:

(i) $K$ and $S$ are Choquet simplexes, and every extreme point in $K($ and $S$ ) is a weak peak point; 
(ii) $K$ and $S$ are metrizable, and every extreme point in $K$ (and $S)$ is a weak peak point;

(iii) $\partial K$ and $\partial S$ are closed, and every extreme point in $K$ (and $S)$ is a split face.

The proof under condition (i) contains the idea of isolated points in the second-dual method in [12]. Noting that $C(X)$ is an $A(K)$-space for some Bauer simplex $K$, and vice versa, Amir and Cambern's result reads as follows:

If $K$ and $S$ are Bauer simplexes and if there is an isomorphism $\varphi: A(K) \rightarrow A(S)$ with $\|\varphi\|\left\|\varphi^{-1}\right\|<2$, then $\partial K$ and $\partial S$ are homeomorphic.

This can be regarded as a special case of (iii) and indeed, as will be shown, condition (iii) is exactly what is needed in Cambern's arguments [9] to construct the homeomorphism. Finally, if $\mathscr{A}$ is a function algebra with state space $K$, then every extreme point of $K$ is both a weak peak point and a split face, and the extreme boundary $\partial K$ identifies with the Choquet boundary of $\mathscr{A}$. Moreover, the uniform closure of re $\mathscr{A}$ identifies with $A(K)$. Therefore the above results apply to function algebras.

2. Compact convex sets. Let $K$ be a compact convex set in a locally convex space. The extreme boundary $\partial K$ of $K$ is the set of extreme points, with the relative topology. A face $F$ of $K$ is called a split face $\left[1 ;\right.$ p. 133] if there is a face $F^{\prime}$ of $K$ such that every point in $K \backslash\left(F \cup F^{\prime}\right)$ can be uniquely represented as a convex combination of a point in $F$ and a point in $F^{\prime}$. The complementary face $F^{\prime}$ is also split and is the union of all the faces disjoint from $F$. Every face of a triangle is split; more generally, every closed face of a Choquet simplex is split [1;p. 144]. Let $A(K)$ be the Banach space of real continuous affine functions on $K$ with the supremum norm and pointwise ordering. The constant function 1 on $K$ is also denoted by 1. We will identify $K$, via the evaluation map, with the state space $\left\{\mu \in A(K)^{*}: \mu(1)=1=\|\mu\|\right\}$ in $A(K)^{*}$ and so, if $F$ is a closed split face of $K$, then $A(K)^{*}=\operatorname{lin} F \oplus_{\ell_{1}} \operatorname{lin} F^{\prime}$ where lin denotes the linear span. Note that the convex hull of a finite number of split faces is also a split face. If $F$ is a closed split face of $K$, then any continuous affine function on $F$ has a norm preserving extension to a continuous affine function on $K$. Moreover, if the complementary face $F^{\prime}$ is also closed, then given $a \in A(F)$ and $b \in A\left(F^{\prime}\right)$, there is an extension $f \in A(K)$ with $f \mid F=a$ and $f \mid F^{\prime}=b$. Let $F$ be a closed split face of $K$ and let $\chi_{F}$ be the characteristic function of $F$. Then the upper 
envelope function

$$
\hat{\chi}_{F}(x)=\inf \left\{a(x): a \in A(K), a>\chi_{F}\right\}
$$

is upper semi-continuous with $F=\hat{\chi}_{F}^{-1}(1)$ and $F^{\prime}=\hat{\chi}_{F}^{-1}(0)$; moreover, the set $\left\{a \in A(K): a>\chi_{F}\right\}$ is directed downward [1; p. 141] and so $\hat{\chi}_{F}$ is affine, and there is a decreasing net $\left(a_{\alpha}\right)$ in $A(K)$, with $a_{\alpha}>\chi_{F}$, converging pointwise to $\hat{\chi}_{F}$ such that for any $\delta>0$, there exists $\alpha_{0}$ such that $\alpha \geq \alpha_{0}$ implies $1+\delta>a_{\alpha}$. These facts are crucial and will be used repeatedly in the sequel. We refer to [1] for further results on compact convex sets and Choquet theory as well as undefined terminology.

Definition. Let $K$ be a compact convex set and let $x \in \partial K$. We call $x$ a weak peak point if given $1>\varepsilon>0$ and an open set $U$ containing $x$, there exists $h \in A(K)$ such that $\|h\| \leq 1, h(x)>1-\varepsilon$, and $|h|<\varepsilon$ on $\partial K \backslash U$.

Proposition 1. If $x$ is a weak point in $K$, then $\{x\}$ is a split face of $K$. The converse holds if $\partial K$ is closed in $K$.

Proof. The first assertion is an extension of Asimow's result in [3]. Write $\chi_{x}$ for the upper envelope $\hat{\chi}_{\{x\}}$ which is concave and u.s.c. Since $x \in \partial K$, we have $K=\operatorname{co}(\{x\} \cup F)$ where $\{x\}=\chi_{x}^{-1}(1)$ and $F=\chi_{x}^{-1}(0)$, and every $z \in K$ has a representation

$$
z=\chi_{x}(z) x+\left(1-\chi_{x}(z)\right) y \quad(y \in F) .
$$

To show that $\{x\}$ is a split face, we need to show that $F$ is convex and the above representation is unique.

We first show that for $1>\varepsilon>0$ and $y_{1}, y_{2}, y_{3} \in F$, there exists $h \in A(K)$ such that $h(x)>1-\varepsilon$ and $\left|h\left(y_{j}\right)\right| \leq \varepsilon$ for $j=1,2,3$. Let $\mu_{j}$ be a maximal measure on $K$ representing $y_{j}$. Then $\mu_{j}\{x\}=$ $\mu_{j}\left(\chi_{\{x\}}\right) \leq \hat{\chi}_{\{x\}}\left(y_{j}\right)=0$ (cf. [1; Proposition I.3.1]). So there exists a closed neighborhood $U_{j}$ of $x$ with $\mu_{j}\left(U_{j}\right)<\frac{\varepsilon}{2}$. Let $U=U_{1} \cap U_{2} \cap U_{3}$. By hypothesis, there exists $h \in A(K)$ such that $\|h\| \leq 1, h(x)>1-\varepsilon$, and $|h|<\frac{\varepsilon}{2}$ on $\partial K \backslash U$. So $|h| \leq \frac{\varepsilon}{2}$ on $\overline{\partial K \backslash U}$ which contains $\overline{\partial K \backslash U}$. By maximality of $\mu_{j}$, we have

$$
\left|h\left(y_{j}\right)\right|=\left|\int_{\overline{\partial K}} h d \mu_{j}\right| \leq \int_{\overline{\partial K} \cap U}|h| d \mu_{j}+\int_{\overline{\partial K} \backslash U}|h| d \mu_{j} \leq \frac{\varepsilon}{2}+\frac{\varepsilon}{2} .
$$

We now show that $F$ is convex. Let $y_{1}, y_{2} \in F$ and let $z=$ $\lambda y_{1}+(1-\lambda) y_{2}$ where $0 \leq \lambda \leq 1$. It suffices to show $\chi_{x}(z)=0$. 
Suppose $\chi_{x}(z)>0$. Write $z=\chi_{x}(z) x+\left(1-\chi_{x}\right)(z) y_{3}$ with $y_{3} \in F$. Let $\varepsilon=\frac{1}{2} \chi_{x}(z)$. Then by the above, there exists $h \in A(K)$ such that $h(x)>1-\varepsilon$ and $\left|h\left(y_{j}\right)\right| \leq \varepsilon$ for $j=1,2,3$. So $|h(z)|=\mid \lambda h\left(y_{1}\right)+$ $(1-\lambda) h\left(y_{2}\right) \mid \leq \varepsilon$. But also $|h(z)|=\left|\chi_{x}(z) h(x)+\left(1-\chi_{x}\right)(z) h\left(y_{3}\right)\right|>$ $\chi_{x}(z)(1-\varepsilon)-\left(1-\chi_{x}\right)(z) \varepsilon=\varepsilon$ which is a contradiction. So $\chi_{x}(z)=0$.

Finally, given any representation $z=\beta x+(1-\beta) y^{\prime}$ with $y^{\prime} \in F$ (and $0<\beta<1), \chi_{x}(z) \geq \beta$ as $\chi_{x}$ is concave. Suppose $\chi_{x}(z)>\beta$. Then equating the two representations yields

$$
y^{\prime}=\frac{\chi_{x}(z)-\beta}{1-\beta}+\frac{1-\chi_{x}(z)}{1-\beta} y
$$

from which $0=\chi_{x}\left(y^{\prime}\right) \geq \frac{\chi_{x}(z)-\beta}{1-\beta}>0$ which is impossible. So $\chi_{x}(z)=$ $\beta$ and it follows that $y^{\prime}=y$.

Now suppose $\partial K$ is closed and $\{x\}$ is a split face. We show that $x$ is a weak peak point. Let $1>\varepsilon>0$ and let $U$ be an open set containing $x$. As $x \in \partial K$, we have $\{x\} \cap \overline{c o}(K \backslash U)=\varnothing$. Let $G=\overline{c o}(K \backslash U)$ and let $F$ be the complementary face of $\{x\}$. Since $\{x\}$ is a split face, there is a decreasing net $\left(a_{\alpha}\right)$ in $A(K)$, with $1+\frac{\varepsilon}{2}>$ $a_{\alpha}>\chi_{x}$, converging pointwise to $\chi_{x}$. Since $G \cap \partial K$ is a compact subset of $F$ and $\chi_{x}=0$ on $F$, we have $a_{\alpha} \downarrow 0$ on $G \cap \partial K$ and by Dini's theorem, we can find $a_{\alpha_{0}} \in A(K)$, with $1+\frac{\varepsilon}{2}>a_{\alpha_{0}}>\chi_{F}$ and $\left|a_{\alpha_{0}}\right|<\frac{\varepsilon}{2}$ on $G \cap \partial K$ which contains $\partial K \backslash U$. Let $h=a_{\alpha_{0}}-\frac{\varepsilon}{2}$. Then $\|h\| \leq 1, h(x)>1-\varepsilon$ and $|h|<\varepsilon$ on $\partial K \backslash U$.

EXAMPLE 1. Every extreme point of a Bauer simplex is a weak peak point.

EXAMPLE 2. Let $K$ be the state space of a function algebra $\mathscr{A}$. Then $\partial K$ identifies with the Choquet boundary Ch $\mathscr{A}$ of $\mathscr{A}$ and every $x \in \partial K$ is a weak peak point by Bishop's characterization of Ch $\mathscr{A}$ (cf. [8; p. 97]). Indeed, our definition originates from this example, and we also note that, as in [8; Theorem 2.3.4], an equivalent definition for a weak peak point $x$ in $K$ is that given $\varepsilon>0$ and open set $U$ containing $x$, there is an $h$ in $A(K)$ such that $\|h\|=1=h(x)$ and $|h|<\varepsilon$ on $\partial K \backslash U$.

Example 3. Let $K$ be the Poulsen simplex (cf. [4; p. 130]). Then every $x \in \partial K$ is a split face, but it is not a weak peak point. Consider $y=\frac{1}{3} x+\frac{2}{3} x^{\prime}$ for some $x^{\prime} \in \partial K \backslash\{x\}$. Choose a closed convex neighborhood $U$ of $x$ such that $y \notin U$. Then $x^{\prime} \notin U$. If $x$ was a weak peak point, there would exist $h \in A(K)$ with $\|h\| \leq 1$, $h(x)>1-\frac{1}{7}$ and $|h|<\frac{1}{7}$ on $\partial K \backslash U$. So $h(y)=\frac{1}{3} h(x)+\frac{2}{3} h\left(x^{\prime}\right)>\frac{4}{21}$. 
Let $y=\lim _{\beta} x_{\beta}$ with $x_{\beta} \in \partial K$. Then $y \notin U$ implies $x_{\beta} \notin U$ eventually and so $h(y)=\lim _{\beta} h\left(x_{\beta}\right) \leq \frac{1}{7}$ which is impossible.

3. Isomorphisms of $A(K)$. Let $K$ be a compact convex set identified with the state space $\left\{\mu \in A(K)^{*}: \mu(1)=1=\|\mu\|\right\}$. Then the convex hull $\operatorname{co}(K \cup-K)$ is the closed unit ball in $A(K)^{*}$ and so $A(K)^{* *}$ identifies naturally with the space $A^{b}(K)$ of bounded affine functions on $K$, with the supremum norm and pointwise ordering. Let $K^{b}=\left\{\mu \in A^{b}(K)^{*}: \mu(1)=1=\|\mu\|\right\} \subset A(K)^{* * *}$. Then $A^{b}(K)$ is isometrically order-isomorphic to $A\left(K^{b}\right)$ and hence we can turn the bounded affine functions on $K$ into weak ${ }^{*}$ continuous affine functions on $K^{b}$. The identifications $A(K)^{* *}=A^{b}(K)=A\left(K^{b}\right)$ will be used throughout. Note that $K$ embeds as a $\sigma\left(A(K)^{* * *}, A(K)^{* *}\right)$-dense convex subset of $K^{b}$, via the embedding $A(K)^{*} \rightarrow A(K)^{* * *}$. Moreover, if $K$ is a Choquet simplex, then $K^{b}$ is a Bauer simplex and $K$ is even a split face of $K^{b}$ [14; Example 3.3(b)]. An important element in Cohen's second-dual method [12] involves the isolated points of $\partial K^{b}$ which turn out to be in $\partial K$.

LEMMA 2. Let $K$ be a compact convex set and let $F$ be a dense face in $K$. If $x \in \partial K$ is an isolated point in $\partial K$, then $x \in F$.

Proof. There is a closed set $G \subset K$ with $\partial K \backslash\{x\}=\partial K \cap G$. Since $x \notin G$, we have $x \notin \overline{c o} G$ by Milman's theorem. By density of $F$, there exists $y \in F$ with $y \notin \overline{\operatorname{co}} G$. Note that $K=\operatorname{co}(\{x\} \cup \overline{c o} G)$ and so $y=\lambda x+(1-\lambda) z$ with $0<\lambda \leq 1$. Now $F$ is a face and $y \in F$ gives $x \in F$.

LEMMA 3. Let $K$ be a compact convex set and consider $K \subset K^{b}$. Let $x \in \partial K$ be a split face of $K$. Then $x$ is an isolated point in $\partial K^{b}$ as well as a split face of $K^{b}$ with a closed complementary face.

Proof. Consider the affine function $\chi_{x} \in A^{b}(K)$ with $\{x\}=\chi_{x}^{-1}(1)$ and $x_{x}^{-1}(0)=\{x\}^{\prime}$ being the complementary face in $K$. Now consider $\chi_{x} \in A\left(K^{b}\right)$ and let $Z$ denote $\left\{z \in K^{b}: \chi_{x}(z)=0\right\}$, a (weak $\left.{ }^{*}\right)$ closed face of $K^{b}$. Let $y \in K^{b} \backslash Z$. By denseness of $K$ in $K^{b}$, $y$ is the weak limit of a net $y_{\alpha}$ in $K \backslash Z$. Since $x$ is a split face of $K, y_{\alpha}=r_{\alpha} x+\left(1-r_{\alpha}\right) z_{\alpha}$ for each $\alpha$ where $z_{\alpha} \in Z \cap K$ and $r_{\alpha}=\chi_{x}\left(y_{\alpha}\right)$. By compactness of $K^{b}$, we may assume $z_{\alpha}$ converges to an element $z$ in $Z$. Taking limits and using the continuity of $\chi_{x}$ on $K^{b}$, we get $y=r x+(1-r) z$ where $r=\chi_{x}(y)$. Consequently, the representation of $y$ as a convex combination of $x$ and 
an element of $Z$ is unique, $x \in \partial K^{b}$, and $\partial K^{b} \backslash\{x\} \subseteq Z$. Hence, $\{x\}=\left\{y \in K^{b} \mid \chi_{x}(y)>\frac{1}{2}\right\} \cap \partial K^{b}$ and $x$ is isolated in $K$.

We thank the referee for pointing out that the following result is accessible via the general results of $[6,7,17]$. Since this result is not stated explicitly in these references, we include here, for completeness, our alternative argument involving isolated points for the interested reader.

THEOREM 4. Let $K$ and $S$ be Choquet simplexes in which every extreme point is a weak peak point. Let $\varphi: A(K) \rightarrow A(S)$ be a surjective linear isomorphism with $\|\varphi\|\left\|\varphi^{-1}\right\|<2$. Then $\partial K$ and $\partial S$ are homeomorphic.

Proof. Consider the second dual map $\varphi^{* *}: A(K)^{* *} \rightarrow A(S)^{* *}$. By previous remarks, and since $K^{b}$ is a Bauer simplex, $A(K)^{* *}=A\left(K^{b}\right)$ $=C\left(\partial K^{b}\right)$; the same applies to $A(S)^{* *}$. Consulting Lemma 3 in [12], the condition $\left|\left(\varphi^{* *} \chi_{x}\right)(y)\right|>\frac{1}{2}\|\varphi\|$ establishes a 1-1 correspondence between the isolated points $x$ of $\partial K^{b}$ and the isolated points $y$ of $\partial S^{b}$. By Lemma 2 and Lemma 3, $\partial K$ is the set of isolated points of $\partial K^{b}$ and the same for $\partial S$. Thus we have a bijection $H: \partial S \rightarrow \partial K$ given by $H(y)=x$ whenever $\left|\left(\varphi^{* *} \chi_{x}\right)(y)\right|>\frac{1}{2}\|\varphi\|$. But this bijection is just the map $\rho$ defined below and, by the weak peak point property, shown to be a homeomorphism in the proof of Theorem 7 .

We now turn to arbitrary compact convex sets $K$ and $S$ in which every extreme point is a split face. Suppose there is an isomorphism $\varphi: A(K) \rightarrow A(S)$ with $\|\varphi\|\left\|\varphi^{-1}\right\|<2$. We may, and will always assume, that $\|\varphi\|<2$ and $\|\varphi(a)\|>c\|a\|$ for all non-zero $a \in A(K)$ where $1<c<2$. This loses no generality for, if necessary, we can replace $\varphi$ by $c\left\|\varphi^{-1}\right\| \varphi$ where $\|\varphi\|\left\|\varphi^{-1}\right\|<\frac{2}{c}<2$. Given such an isomorphism, how does one construct a homeomorphism $\rho: \partial S \rightarrow$ $\partial K$ ? We follow Cambern's approach. Let $y \in \partial S$. Consider the dual map $\varphi^{*}: A(S)^{*} \rightarrow A(K)^{*}$. Since every $x \in \partial K$ is a split face of $K \subset A(K)^{*}$, we can write $\varphi^{*}(y)=\lambda x+\mu$ for some $\lambda \in \mathbb{R}$ and $\mu \in \operatorname{lin}\{x\}^{\prime}$, where $\{x\}^{\prime}$ is the complementary face. It turns out that, if $K$ and $S$ are metrizable, then there is a unique $x \in \partial K$ for which $|\lambda|$ is large, that is, $|\lambda|>c$. So we can define $\rho(y)=x$ and show it is a bijection which is even a homeomorphism if every extreme point is a weak peak point. In the nonmetrizable non-simplex case, however, we need to assume the closedness of the extreme boundaries to complete 
the arguments. Note that, in the classical Banach-Stone theorem, $\varphi$ is an isometry with $\lambda=1$ and $\mu=0$, and $\varphi^{*}$ itself already induces a homeomorphism.

Now we describe the details. Let $x \in \partial K$ and let $\chi_{x}=\hat{\chi}_{\{x\}}$ be the upper envelope function which is affine and u.s.c. on $K$. Consider $\chi_{x} \in A(K)^{* *}$. We will denote by $\langle\cdot, \cdot\rangle$ the bilinear functional on a pair of Banach spaces in duality. Let $y \in \partial S$ with $\varphi^{*}(y)=\lambda x+\mu$ as in the previous paragraph. Then, noting that $\{x\}=\chi_{x}^{-1}(1)$ and $\{x\}^{\prime}=$ $\chi_{x}^{-1}(0)$, we have $\left\langle y, \varphi^{* *} \lambda \chi_{x}\right\rangle=\left\langle\varphi^{*}(y), \chi_{x}\right\rangle=\lambda\left\langle x, \chi_{x}\right\rangle+\left\langle\mu, \chi_{x}\right\rangle=\lambda$. As in [9], we have the following basic contructions. Let

$$
Y=\left\{y \in \partial S: \exists x \in \partial K \text { with }\left|\left\langle y, \varphi^{* *} \chi_{x}\right\rangle\right|>c\right\} .
$$

First observe that for each $y \in Y$, there is at most one $x \in \partial K$ with $\left|\left\langle y, \varphi^{* *} \chi_{x}\right\rangle\right|>c$. Indeed, if there are $x, x^{\prime} \in \partial K$ with $\varphi^{*}(y)=\lambda x+$ $\mu=\lambda^{\prime} x^{\prime}+\mu^{\prime}$ and $|\lambda|,\left|\lambda^{\prime}\right|>c$, then $2>\left\|\varphi^{*}(y)\right\|=|\alpha|+\|\mu\|>c+\|\mu\|$ implies $2-c>\|\mu\|$ which gives $(2-c)+(2-c)>\|\mu\|+\left\|\mu^{\prime}\right\| \geq$ $\left\|\mu-\mu^{\prime}\right\|=\left\|\lambda^{\prime} x^{\prime}-\lambda x\right\|=\left|\lambda^{\prime}\right|+|\lambda|>2 c$, contradicting $c>1$. Therefore, we can define $\rho: Y \rightarrow \partial K$ by $\rho(y)=x$ whenever $\left|\left\langle y, \varphi^{* *} \chi_{x}\right\rangle\right|>c$. Likewise, we let

$$
X=\left\{x \in \partial K: \exists y \in \partial S \text { with }\left|\left\langle x, \varphi^{* *-1} \chi_{y}\right\rangle\right|>\frac{1}{2}\right\}
$$

and define $\tau: X \rightarrow \partial S$ by $\tau(x)=y$ whenever $\left|\left\langle x, \varphi^{* *-1} \chi_{y}\right\rangle\right|>\frac{1}{2}$. What remains is to show that $Y=\partial S$ and $\rho$ is a homeomorphism. We demonstrate this for the metrizable and the nonmetrizable cases separately in the next two sections.

4. The metrizable case. We assume in this section that $K$ and $S$ are metrizable, that every extreme point of $K$ (and $S$ ) is a split face in the following two lemmas. We adopt the previous notations.

\section{Lemma 5. The maps $\rho: Y \rightarrow \partial K$ and $\tau: X \rightarrow \partial S$ are onto.}

Proof. Let $x \in \partial K$. We show there exists $y \in \partial S$ with $\left|\left\langle y, \varphi^{* *} \chi_{x}\right\rangle\right|$ $>c$. By separability of $A(K)$, there is a decreasing sequence $\left(a_{n}\right)$ in $A(K)$ converging to $\chi_{x}$ pointwise. So $\varphi^{* *} \chi_{x}=\lim _{n} \varphi\left(a_{n}\right)$ pointwise on $S$. If $\left|\left\langle y, \varphi^{* *} \chi_{x}\right\rangle\right| \leq c$ for all $y \in \partial S$, then $\left|\left\langle y, \lim _{n} \varphi\left(a_{n}\right)\right\rangle\right| \leq c$ for all $y \in \partial S$ and by Meyer's maximum principle [1; Proposition I.4.10], we have

$$
\begin{aligned}
c \geq \sup _{y \in S}\left|\left\langle y, \lim _{n} \varphi\left(a_{n}\right)\right\rangle\right| & =\sup _{y \in S}\left|\left\langle y, \varphi^{* *} \chi_{x}\right\rangle\right| \\
& =\left\|\varphi^{* *} \chi_{x}\right\|>c\left\|\chi_{x}\right\|=c
\end{aligned}
$$


which is a contradiction. So $Y$ is nonempty and $\rho$ is onto. Similarly $\tau$ is onto.

LEMMA 6. If $y \in Y$ with $\rho(y)=x$, then $x \in X$ and $\tau(x)=y$. Hence $Y=\partial S$ and $\rho: \partial S \rightarrow \partial K$ is a bijection.

Proof. Suppose either $x \notin X$ or $x \in X$ but $\tau(x) \neq y$. Then $\left|\left\langle x, \varphi^{* *-1} \chi_{y}\right\rangle\right| \leq \frac{1}{2}$. We deduce a contradiction. Let

$$
d=\sup _{x^{\prime} \in \partial K}\left|\left\langle x^{\prime}, \varphi^{* *-1} \chi_{y}\right\rangle\right|
$$

Since $\chi_{y}$ is the pointwise limit of a sequence of continuous affine functions, as in Lemma 5, we have $\left\|\varphi^{* *-1} \chi_{y}\right\| \leq d$ by Meyer's maximum principle. Note that $d>\frac{d}{c}$ and by Lemma $5, d>\frac{1}{2}$. So we can pick $x^{\prime} \in \partial K$ such that $\left|\left\langle x^{\prime}, \varphi^{* *-1} \chi_{y}\right\rangle\right|>\frac{d}{c}, \frac{1}{2}$. There exists $y^{\prime} \in Y$ with $\rho\left(y^{\prime}\right)=x^{\prime}$. As $\left|\left\langle x, \varphi^{* *-1} \chi_{y}\right\rangle\right| \leq \frac{1}{2}, x \neq x^{\prime}$ and so $y \neq$ $y^{\prime}$. Therefore $0=\left\langle y^{\prime}, \chi_{y}\right\rangle=\left\langle\varphi^{*}\left(y^{\prime}\right), \varphi^{* *-1} \chi_{y}\right\rangle=\left\langle\lambda^{\prime} x^{\prime}, \varphi^{* *-1} \chi_{y}\right\rangle+$ $\left\langle\mu^{\prime}, \varphi^{* *-1} \chi_{y}\right\rangle$ where, as before, $\varphi^{*}\left(y^{\prime}\right)=\lambda^{\prime} x^{\prime}+\mu^{\prime}$ with $\left|\lambda^{\prime}\right|>c$ and $\left\|\mu^{\prime}\right\|<2-c<1$. Now $\left|\left\langle\lambda^{\prime} x^{\prime}, \varphi^{* *-1} \chi_{y}\right\rangle\right|>\left|\lambda^{\prime}\right| \cdot \frac{d}{c}>d$ while $\left|\left\langle\mu^{\prime}, \varphi^{* *-1} \chi_{y}\right\rangle\right| \leq\left\|\mu^{\prime}\right\|\left\|\varphi^{* *-1} \chi_{y}\right\|<(2-c) d<d$. This is impossible. The last conclusion can be easily verified, using Lemma 5.

Finally, to prove that $\rho$ is a homeomorphism, we need to assume a slightly stronger condition, namely, that every extreme point is a weak peak point.

TheOREM 7. Let $K$ and $S$ be metrizable compact convex sets in which every extreme point is a weak peak point. If there exists an isomorphism $\varphi: A(K) \rightarrow A(S)$ with $\|\varphi\|\left\|\varphi^{-1}\right\|<2$, then $\partial K$ and $\partial S$ are homeomorphic.

Proof. We show that the bijection $\rho: \partial S \rightarrow \partial K$ is a homeomorphism. Let $F \subset \partial K$ be closed and let $F=\partial K \cap G$ for some closed set $G \subset K$. We show $\rho^{-1}(F)$ is closed in $\partial S$. Let $x \in \partial K \backslash F$ with $\rho(y)=x$. Then $y \notin \rho^{-1}(F)$ and there is a closed neighborhood $V$ of $x$ such that $V \cap G=\varnothing$. Note that $F \subset \partial K \backslash V$. As before, write $\varphi^{*}(y)=\lambda x+\mu$ with $|\lambda|>c$ and $\mu \in \operatorname{lin}\{x\}^{\prime}$. Write $\mu=\sum_{i=1}^{n} r_{i} k_{i}$ with $r_{i} \in \mathbb{R}$ and $k_{i} \in\{x\}^{\prime}$. Let $r=\sum_{i=1}^{n}\left|r_{i}\right|$ and let $\mu_{j}$ be a maximal measure on $K$ representing $k_{j}$. Choose $1>\varepsilon>0$ such that $\varepsilon<\min \left(\frac{|\lambda|-c}{r+|\lambda|}, c-1\right)$. Since $x$ is a weak peak point, as in the proof of Proposition 1, there are closed neighborhoods $U_{j}$ of $x$ with $\mu_{j}\left(U_{j}\right)<\frac{\varepsilon}{2}$, and if we let $U=U_{1} \cap \cdots \cap U_{n} \cap V$ and noting that 
$F \subset \partial K \backslash U$, we can find $h_{x} \in A(K)$ such that $\left\|h_{x}\right\| \leq 1, h_{x}(x)>1-\varepsilon$ and $\left|h_{x}\right| \leq \varepsilon$ on $F \cup\left\{k_{1}, \ldots, k_{n}\right\}$. We claim

$$
\rho^{-1}(F)=\bigcap_{x \in \partial K \backslash F}\left\{y \in \partial S:\left|\left\langle\varphi\left(h_{x}\right), y\right\rangle\right| \leq c\right\} .
$$

Indeed $y \notin \rho^{-1}(F)$ implies $\left|\left\langle\varphi\left(h_{x}\right), y\right\rangle\right|=\left|\left\langle h_{x}, \varphi^{*}(y)\right\rangle\right|=\mid\left\langle h_{x}, \lambda x\right\rangle+$ $\left\langle h_{x}, \mu\right\rangle|\geq| \lambda \mid(1-\varepsilon)-r \varepsilon>c$ since $\left|\left\langle h_{x}, \mu\right\rangle\right|=\left|\sum_{i=1}^{n}\left\langle h_{x}, r_{i} k_{i}\right\rangle\right| \leq$ $\sum_{i=1}^{n}\left|r_{i}\right| \varepsilon=r \varepsilon$. Also, for $y^{\prime} \in \rho^{-1}(F)$ with $\varphi^{*}\left(y^{\prime}\right)=\lambda^{\prime} x^{\prime}+\mu^{\prime}$, $\left|\lambda^{\prime}\right|>c$ and $x^{\prime} \in F$, we have $\left|\left\langle\varphi\left(h_{x}\right), y^{\prime}\right\rangle\right|=\left|\left\langle h_{x}, \lambda^{\prime} x^{\prime}\right\rangle+\left\langle h_{x}, \mu^{\prime}\right\rangle\right| \leq$ $\left|\lambda^{\prime}\right| \varepsilon+\left\|\mu^{\prime}\right\|<2 \varepsilon+(2-c)<c$. So $\rho^{-1}(F)$ is closed in $\partial S$ and $\rho$ is continuous. Similar arguments show that $\rho^{-1}$ is continuous.

5. The nonmetrizable case. From now on, $K$ and $S$ are nonmetrizable compact convex sets in which every extreme point is a split face. We assume that $\partial K$ and $\partial S$ are closed in $K$ and $S$ respectively. As before, let $\varphi: A(K) \rightarrow A(S)$ be an isomorphism such that $\|\varphi\|<2$ and $\|\varphi(a)\|>c\|a\|$ with $1<c<2$. Then one can define the maps $\rho: Y \rightarrow \partial K$ and $\tau: X \rightarrow \partial S$. We need to show $Y=\partial S$ and $\rho$ is a homeomorphism. In the metrizable case, this can be achieved by invoking Meyer's maximum principle which, however, is not available in the nonmetrizable case. Instead, one needs more delicate arguments.

Our first task is to show that $Y \neq \varnothing$ and $\rho$ is onto, in other words, given $x \in \partial K$, we need to find $y \in \partial S$ such that $\left|\left\langle y, \varphi^{* *} \chi_{x}\right\rangle\right|>c$. Fix a decreasing net $\left(a_{\alpha}\right)$ in $A(K)$ converging pointwise to $\chi_{x}$ such that $a_{\alpha}>\chi_{x}$ and for any $\delta>0$, we have $1+\delta>a_{\alpha}$ from some $\alpha_{0}$ onwards. Then $\varphi^{* *} \lambda_{x}=\lim _{\alpha} \varphi\left(a_{\alpha}\right)$ pointwise on $S$ and so we need to find $y \in \partial S$ with $\left|\left\langle y, \lim _{\alpha} \varphi\left(a_{\alpha}\right)\right\rangle\right|>c$. Fix $x \in \partial K$ as above. For each $\alpha$, let $\partial S_{\alpha}=\left\{y \in \partial S:\left|\left\langle\varphi\left(a_{\alpha}\right), y\right\rangle\right|>c\right\}$. Note that $\partial S_{\alpha} \neq \varnothing$ since $\varphi\left(a_{\alpha}\right) \in A(S)$ and $\sup _{y \in \partial S}\left|\left\langle\varphi\left(a_{\alpha}\right), y\right\rangle\right|=\left\|\varphi\left(a_{\alpha}\right)\right\|>c\left\|a_{\alpha}\right\|>c$. Let $\partial S_{x}=\left\{y \in \partial S: y\right.$ is a cluster point of $\left\{y_{\alpha}\right\}$ with $\left.y_{\alpha} \in \partial S_{\alpha}\right\}$. Since $\partial S_{\alpha} \neq \varnothing$ and since $\partial S$ is compact, we have $\partial S_{x} \neq \varnothing$. Now we are going to show that $\partial S_{x}$ is finite and it contains the point we are looking for.

Lemma 8. Let $1>\varepsilon>0$ and $c-2 \varepsilon>1$. Let $y \in \partial S_{x}$. If $g \in A(S)$ satisfies $g(y)>1-\varepsilon$ and $\|g\| \leq 1$, then $\left|\left\langle\varphi^{-1}(g), x\right\rangle\right| \geq \frac{1}{2}(c-1-2 \varepsilon)$.

Proof. Let $y$ be a cluster point of $\left\{y_{\alpha}\right\}$ with $y_{\alpha} \in \partial S_{\alpha}$. By choosing a subnet, we may assume $1+\frac{\varepsilon}{2}>a_{\alpha}$ and $g\left(y_{\alpha}\right)>1-\varepsilon$ for all $\alpha$. We have $\left|\left\langle\varphi\left(a_{\alpha}\right), y_{\alpha}\right\rangle\right|>c$. Let $\lambda_{\alpha}= \pm 1$ such that $\left\langle\varphi\left(\lambda_{\alpha} a_{\alpha}\right), y_{\alpha}\right\rangle>$ $c$. Then $\left\|g+\varphi\left(\lambda_{\alpha} a_{\alpha}\right)\right\| \geq\left|g\left(y_{\alpha}\right)+\left\langle\varphi\left(\lambda_{\alpha} a_{\alpha}\right), y_{\alpha}\right\rangle\right|>1-\varepsilon+c$. So 
$\left\|\varphi^{-1}(g)+\lambda_{\alpha} a_{\alpha}\right\|>\frac{1}{2}(1-\varepsilon+c)>1$. Note that $\left\|\varphi^{-1}\right\| \leq \frac{1}{c}<1$ and $\left\|\varphi^{-1}(g)\right\|<1$. For each $\alpha$, by Bauer's maximum principle [1; p. 46], there exists $x_{\alpha} \in \partial K$ such that

$$
\left|\left\langle\varphi^{-1}(g), x_{\alpha}\right\rangle+\lambda_{\alpha} a_{\alpha}\left(x_{\alpha}\right)\right|=\left\|\varphi^{-1}(g)+\lambda_{\alpha} a_{\alpha}\right\|>\frac{1}{2}(1-\varepsilon+c)
$$

which implies $\left|\left\langle\varphi^{-1}(g), x_{\alpha}\right\rangle\right|>\frac{1}{2}(1-\varepsilon+c)-\left(1+\frac{\varepsilon}{2}\right)=\frac{1}{2}(c-1-2 \varepsilon)$ and $a_{\alpha}\left(x_{\alpha}\right)>\frac{1}{2}(c-1-\varepsilon)$. By compactness of $\partial K$, we may assume $\left(x_{\alpha}\right)$ converges to some $z \in \partial K$. We show $z=x$. Suppose $z \neq x$. Then $\lim _{\alpha} a_{\alpha}(z)=\chi_{x}(z)=0$. So there exists $\alpha_{1}$ such that $a_{\alpha_{1}}(z)<$ $\frac{1}{2}(c-1-\varepsilon)$. Further, there exists $\alpha \geq \alpha_{1}$ such that $a_{\alpha_{1}}\left(x_{\alpha}\right)<$ $\frac{1}{2}(c-1-\varepsilon)$. As $\left(a_{\alpha}\right)$ is decreasing, we have $\frac{1}{2}(c-1-\varepsilon)<a_{\alpha}\left(x_{\alpha}\right)$ $\leq a_{\alpha_{1}}\left(x_{\alpha}\right)<\frac{1}{2}(c-1-\varepsilon)$ which is a contradiction. So $x_{\alpha} \rightarrow x$ and it follows that $\left|\left\langle\varphi^{-1}(g), x\right\rangle\right|=\lim _{\alpha}\left|\left\langle\varphi^{-1}(g), x_{\alpha}\right\rangle\right| \geq \frac{1}{2}(c-1-2 \varepsilon)$.

\section{LEMMA 9. $\partial S_{x}$ is finite.}

Proof. Let $y_{1}, \ldots, y_{n} \in \partial S_{x}$ with $n>1$. If $c-\frac{2}{n} \leq 1$, then $n \leq \frac{2}{c-1}$ and there is nothing to prove. Let $c-\frac{2}{n}>1$. Pick $g_{1} \in A(S)$ such that $\left\|g_{1}\right\| \leq 1, g_{1}\left(y_{1}\right)=1-\frac{1}{n}$ and $g\left(y_{j}\right)=0$ for $j \neq 1$. This can be done because $\left\{y_{i}\right\}$ is a split face of $\operatorname{co}\left\{y_{i}, \ldots, y_{n}\right\}$ which is in turn a closed split face of $S$. Let $G=\left\{y \in S:\left|g_{1}(y)\right| \geq \frac{1}{n}\right\} \cup$ $\left\{y_{j}: j \neq 2\right\}$ which is closed in $S$. Since $y_{2} \notin G$ and $y_{2}$ is a weak peak point by Proposition 1 , there exists $g_{2} \in A(S)$ with $\left\|g_{2}\right\| \leq 1$, $g_{2}\left(y_{2}\right)>1-\frac{1}{n}$ and $\left|g_{2}\right|<\frac{1}{n}$ on $\partial S \cap G$. Next, consider $y_{3} \notin\{y \in$ $S:\left|g_{1}(y)\right| \geq \frac{1}{n}$ or $\left.\left|g_{2}(y)\right| \geq \frac{1}{n}\right\} \cup\left\{y_{j}: j \neq 3\right\}$, we get $g_{3} \in A(S)$ with $\left\|g_{3}\right\| \leq 1, g_{3}\left(y_{3}\right)>1-\frac{1}{n}$ and $\left|g_{3}\right|<\frac{1}{n}$ on $\left\{y \in \partial S:\left|g_{1}(y)\right| \geq \frac{1}{n}\right.$ or $\left.\left|g_{2}(y)\right| \geq \frac{1}{n}\right\} \cup\left\{y_{j}: j \neq 3\right\}$. Continue. We get $g_{1}, \ldots, g_{n} \in A(S)$ with $\left\|g_{j}\right\| \leq 1 \forall_{j}, g_{j}\left(y_{j}\right)>1-\frac{1}{n}$ and $\left|g_{j}\right|<\frac{1}{n}$ on

$$
\left\{y \in \partial S:\left|g_{k}(y)\right| \geq \frac{1}{n} \text { for some } k \leq j-1\right\} \cup\left\{y_{k}: k \neq j\right\} .
$$

We show that $\left\|g_{1}+\cdots+g_{n}\right\|=\sup _{y \in \partial S}\left|\left(g_{1}+\cdots+g_{n}\right)(y)\right|<2$. Let $y \in \partial S$. If $y=y_{j}$ for some $j$, then $\left|g_{j}\left(y_{j}\right)\right| \leq 1$ and $\left|g_{k}\left(y_{j}\right)\right|<\frac{1}{n}$ for $k \neq j$. So $\left|\left(g_{i}+\cdots+g_{n}\right)\left(y_{j}\right)\right|<1+\frac{n-1}{n}<2$. If $y \neq y_{j}$ for all $j$, then either $\left|g_{j}(y)\right|<\frac{1}{n}$ for all $j \leq n-1$ or $\left|g_{j}(y)\right| \geq \frac{1}{n}$ for some $j \leq n-1$. In the former case, $\left|\left(g_{i}+\cdots+g_{n}\right)(y)\right|<\frac{n-1}{n}+1<2$. In the latter, we have $\left|g_{k}(y)\right|<\frac{1}{n}$ for $k \neq j$ and again

$$
\left|\left(g_{1}+\cdots+g_{n}\right)(y)\right|<\frac{n-1}{n}+1 .
$$


So $\left\|g_{1}+\cdots+g_{n}\right\|<2$. Now by Lemma 8 , either

$$
\left\langle\varphi^{-1}\left(g_{j}\right), x\right\rangle>\frac{1}{2}\left(c-1-\frac{2}{n}\right) \text { or }\left\langle\varphi^{-1}\left(-g_{j}\right) x\right\rangle>\frac{1}{2}\left(c-1-\frac{2}{n}\right) \text {. }
$$

As the norm $\left\|g_{1}+\cdots+g_{n}\right\|$ remains the same if we change the sign of any $g_{j}$, we have, by changing signs if necessary, that

$$
\begin{aligned}
2>\left\|g_{1}+\cdots+g_{n}\right\| & =\| \varphi\left(\varphi^{-1}\left(\sum_{j} g_{j}\right) \|\right. \\
& >\left\|\sum_{j} \varphi^{-1}\left(g_{j}\right)\right\| \\
& \geq\left|\sum_{j}\left\langle\varphi^{-1}\left(g_{j}\right), x\right\rangle\right|>\frac{n}{2}\left(c-1-\frac{2}{n}\right) .
\end{aligned}
$$

So $n<\frac{6}{c-1}$ and $\partial S_{x}$ is finite.

LeMma 10. The map $\rho: Y \rightarrow \partial K$ is onto.

Proof. Let $x \in \partial K$ and let $a_{\alpha} \downarrow \chi_{x}$ as above. By Lemma 9, $\partial S_{x}$ is a finite set $\left\{y_{1}, \ldots, y_{n}\right\}$ say. We show there exists $y_{j} \in \partial S_{x}$ with $\left|\left\langle y_{j}, \varphi^{* *} \chi_{x}\right\rangle\right|=\left|\left\langle y_{j}, \lim _{\alpha} \varphi\left(a_{\alpha}\right)\right\rangle\right|>c$. Suppose, otherwise, $\left|\left\langle y_{j}, \lim _{\alpha} \varphi\left(a_{\alpha}\right)\right\rangle\right| \leq c$ for all $j$. Let $F=\operatorname{co}\left\{y_{1}, \ldots, y_{n}\right\}$ which is a closed split face of $S$. Let $F^{\prime}$ be its complementary face. Recall that $\operatorname{co}(S \cup-S)$ is the closed unit ball in $A(S)^{*}$. Since $\left\|\varphi^{*-1}(x)\right\|<\frac{1}{c}<1$, we can write

$$
\varphi^{*-1}(x)=\lambda_{1} \beta_{1} z_{1}+\lambda_{1}\left(1-\beta_{1}\right) z_{1}^{\prime}-\lambda_{2} \beta_{2} z_{2}-\lambda_{2}\left(1-\beta_{2}\right) z_{2}^{\prime}
$$

where $\lambda_{1}+\lambda_{2}=\left\|\varphi^{*-1}(x)\right\|, 0 \leq \beta_{1}, \beta_{2} \leq 1, z_{1}, z_{2} \in F$ and $z_{1}^{\prime}, z_{2}^{\prime}, \in F^{\prime}$. Let $\delta=\frac{1}{2}\left(1-\left(\lambda_{1}+\lambda_{2}\right) c\right)>0$. There exists $\alpha_{1}$ such that $\alpha \geq \alpha_{1}$ implies $\left|\left\langle\varphi\left(a_{\alpha}\right), y_{j}\right\rangle\right|<c+\delta$ for $j=1, \ldots, n$. Let $\alpha \geq \alpha_{1}$. Then

$$
\begin{aligned}
\left\langle\varphi\left(a_{\alpha}\right), \varphi^{*-1}(x)\right\rangle= & \lambda_{1} \beta_{1}\left\langle\varphi\left(a_{\alpha}\right), z_{1}\right\rangle-\lambda_{2} \beta_{2}\left\langle\varphi\left(a_{\alpha}\right), z_{2}\right\rangle \\
& +\left\langle\varphi\left(a_{\alpha}\right), \lambda_{1}\left(1-\beta_{1}\right) z_{1}^{\prime}-\lambda_{2}\left(1-\beta_{2}\right) z_{2}^{\prime}\right\rangle
\end{aligned}
$$

where $\left|\left\langle\varphi\left(a_{\alpha}\right), z_{i}\right\rangle\right|<c+\delta$ for $i=1,2$. Let $\mu_{1}$ and $\mu_{2}$ be maximal measures on $S$ representing $z_{1}^{\prime}$ and $z_{2}^{\prime}$ respectively. Then the supports of $\mu_{1}$ and $\mu_{2}$ are contained in $F^{\prime}$ by [1; Corollary II.611]. Let $\mu=\lambda_{1}\left(1-\beta_{1}\right) \mu_{1}-\lambda_{2}\left(1-\beta_{2}\right) \mu_{2}$. Then $\mu\left(\partial S_{x}\right)=0$ and $\mu$ is supported by $\partial S$ as $\mu_{1}$ and $\mu_{2}$ are. Therefore there is a compact set 
$G \subset \partial S$ such that $G \cap \partial S_{x}=\varnothing$ and $\mu(\partial S \backslash G)<\frac{\delta}{2}$. As $G \cap \partial S_{x}=\varnothing$, there exists $\alpha_{2}$ such that $\alpha \geq \alpha_{2}$ implies $\left|\left\langle\varphi\left(a_{\alpha}\right), y\right\rangle\right| \leq c$ for all $y \in G$. Note that $|\mu|(G) \leq \lambda_{1}\left(1-\beta_{1}\right)+\lambda_{2}\left(1-\beta_{2}\right)$. It follows that, for $\alpha \geq \alpha_{1}, \alpha_{2}$,

$$
\begin{aligned}
1< & \left\langle\varphi\left(a_{\alpha}\right), \varphi^{*-1}(x)\right\rangle<\lambda_{1} \beta_{1}(c+\delta) \\
& +\lambda_{2} \beta_{2}(c+\delta)+\left|\int_{\partial S} \varphi\left(a_{\alpha}\right) d \mu\right| \\
\leq & \lambda_{1} \beta_{1} c+\lambda_{2} \beta_{2} c+\lambda_{1} \beta_{1} \delta+\lambda_{2} \beta_{2} \delta \\
& +\left|\int_{G} \varphi\left(a_{\alpha}\right) d \mu\right|+\left|\int_{\partial S \backslash G} \varphi\left(a_{\alpha}\right) d \mu\right| \\
\leq & \lambda_{1} \beta_{1} c+\lambda_{2} \beta_{2} c+\lambda_{1} \beta_{1} \delta+\lambda_{2} \beta_{2} \delta \\
& +\lambda_{1}\left(1-\beta_{1}\right) c+\lambda_{2}\left(1-\beta_{2}\right) c+2 \cdot \frac{\delta}{2} \\
= & \left(\lambda_{1} \beta_{1}+\lambda_{2} \beta_{2}\right) \delta+\left(\lambda_{1}+\lambda_{2}\right) c+\delta \\
= & \frac{1}{2}\left(1-\left(\lambda_{1}+\lambda_{2}\right) c\right)\left(1+\lambda_{1} \beta_{1}+\lambda_{2} \beta_{2}\right)+\left(\lambda_{1}+\lambda_{2}\right) c \\
< & 1-\left(\lambda_{1}+\lambda_{2}\right) c+\left(\lambda_{1}+\lambda_{2}\right) c=1
\end{aligned}
$$

which is a contradiction. So there exists $y_{j} \in \partial S_{x}$ with $\left|\left\langle y_{j}, \varphi^{* *} \chi_{x}\right\rangle\right|>$ $c$. This proves $Y \neq \varnothing$ and $\rho$ is onto. Analogously, one can show that $\tau: X \rightarrow \partial S$ is onto by considering, for each fixed $y \in \partial S$, the finite set $\partial K_{y}$ of cluster points of $\left\{x_{\alpha}\right\}$ where $x_{\alpha} \in \partial K_{\alpha}=\{x \in$ $\left.\partial K:\left|\left\langle\varphi^{-1}\left(b_{\alpha}\right), x\right\rangle\right|>\frac{1}{2}\right\}$ and $\left(b_{\alpha}\right)$ is a decreasing net in $A(S)$ converging pointwise to $\chi_{y} \in A^{b}(S)$ and for any $\delta>0, b_{\alpha}<1+\delta$ for large $\alpha$. As before, the following lemma shows that $\rho: \partial S \rightarrow \partial K$ is a bijection.

LEMMA 11. If $y \in Y$ with $\rho(y)=x$, then $x \in X$ and $\tau(x)=y$.

Proof. Suppose either $x \notin X$ or $x \in X$ but $\tau(x) \neq y$. We deduce a contradiction. Let $\left(b_{\alpha}\right)$ be as above and let $\partial K_{y}=\left\{x_{1}, \ldots, x_{n}\right\} \subset$ $\partial K$ with $\left|\left\langle x_{1}, \lim _{\alpha} \varphi^{-1}\left(b_{\alpha}\right)\right\rangle\right|>\frac{1}{2}$. Let $0<\varepsilon<\frac{c-1}{2}$. There exists $\alpha_{1}$ such that $\alpha \geq \alpha_{1}$ implies $\left|\left\langle\varphi^{-1}\left(b_{\alpha}\right), x_{1}\right\rangle\right|>\frac{1}{2}-\varepsilon$. As $\left|\left\langle x, \lim _{\alpha} \varphi^{-1}\left(b_{\alpha}\right)\right\rangle\right| \leq \frac{1}{2}$, we have $x \neq x_{1}$ and so $y \neq y_{1}$ where $\rho\left(y_{1}\right)=x_{1}$. Consider $\varphi^{*}\left(y_{1}\right)=\sigma x_{1}+\nu$ with $|\sigma|>c$ and $\|\nu\|<$ $2-c<1$. Let $F=\operatorname{co}\left\{x_{2}, \ldots, x_{n}\right\}$ which is a split face of $K$. Now $\left|\left\langle x_{j}, \lim _{\alpha} \varphi^{-1}\left(b_{\alpha}\right)\right\rangle\right| \leq \frac{1}{2}$ for $j=2, \ldots, n$, and as in the proof of Lemma 10 , we can write

$$
\nu=\lambda_{1} \beta_{1} z_{1}+\lambda_{1}\left(1-\beta_{1}\right) z_{1}^{\prime}-\lambda_{2} \beta_{2} z_{2}-\lambda_{1}\left(1-\beta_{2}\right) z_{2}^{\prime}
$$


where $\lambda_{1}+\lambda_{2}=\|\nu\|<2-c, 0 \leq \beta_{1}, \beta_{2} \leq 1, z_{1}, z_{2} \in F$ and $z_{1}^{\prime}, z_{2}^{\prime}$ lie in the complementary face $F^{\prime}$. Moreover, as in that proof, by constructing a representing measure $\mu$ and a compact set $G \subset \partial K$ with $\mu(\partial K \backslash G)<\varepsilon$, one can show that, for large $\alpha$,

$$
\begin{aligned}
\mid\left\langle\varphi^{-1}\right. & \left.\left(b_{\alpha}\right), \nu\right\rangle \mid \\
< & \lambda_{1} \beta_{1}\left(\frac{1}{2}+\varepsilon\right)+\lambda_{2} \beta_{2}\left(\frac{1}{2}+\varepsilon\right) \\
& +\left|\left\langle\varphi^{-1}\left(b_{\alpha}\right), \lambda_{1}\left(1-\beta_{1}\right) z_{1}^{\prime}-\lambda_{2}\left(1-\beta_{2}\right) z_{2}^{\prime}\right\rangle\right| \\
\leq & \lambda_{1} \beta_{1}\left(\frac{1}{2}+\varepsilon\right)+\lambda_{2} \beta_{2}\left(\frac{1}{2}+\varepsilon\right) \\
& +\left|\int_{G} \varphi^{-1}\left(b_{\alpha}\right) d \mu\right|+\left|\int_{\partial K \backslash G} \varphi^{-1}\left(b_{\alpha}\right) d \mu\right| \\
\leq & \lambda_{1} \beta_{1}\left(\frac{1}{2}+\varepsilon\right)+\lambda_{2} \beta_{2}\left(\frac{1}{2}+\varepsilon\right)+\frac{1}{2} \lambda_{1}\left(1-\beta_{1}\right)+\frac{1}{2} \lambda_{2}\left(1-\beta_{2}\right)+\varepsilon \\
= & \frac{1}{2}\left(\lambda_{1}+\lambda_{2}\right)+\left(\lambda_{1}+\lambda_{2}\right) \varepsilon<(2-c)\left(\frac{1}{2}+\varepsilon\right)
\end{aligned}
$$

Since $y \neq y_{1}$, there exists $b \in A(S)$ such that $b>\chi_{y}$ and $b\left(y_{1}\right)=0$ and it follows that $b_{\alpha}\left(y_{1}\right)=0$ for large $\alpha$. Hence $0=b_{\alpha}\left(y_{1}\right)=$ $\left\langle\varphi^{-1}\left(b_{\alpha}\right), \varphi^{*}\left(y_{1}\right)\right\rangle=\left\langle\varphi^{-1}\left(b_{\alpha}\right), \sigma x_{1}\right\rangle+\left\langle\varphi^{-1}\left(b_{\alpha}\right), \nu\right\rangle$ where

$$
\left|\left\langle\varphi^{-1}\left(b_{\alpha}\right), \sigma x_{1}\right\rangle\right|>c\left(\frac{1}{2}-\varepsilon\right)>(2-c)\left(\frac{1}{2}+\varepsilon\right)>\left|\left\langle\varphi^{-1}\left(b_{\alpha}\right), \nu\right\rangle\right|
$$

which is a contradiction. This proves the lemma.

Finally, the proof for $\rho: \partial S \rightarrow \partial K$ being a homeomorphism is as in the metrizable case and so we obtain the following result.

THEOREM 12. Let $K$ and $S$ be compact convex sets with closed extreme boundaries $\partial K$ and $\partial S$ respectively. Let every extreme point of $K$ (and $S$ ) be a split face. If there is an isomorphism $\varphi: A(K) \rightarrow$ $A(S)$ with $\|\varphi\|\left\|\varphi^{-1}\right\|<2$, then $\partial K$ and $\partial S$ are homeomorphic.

We end with some examples and applications to function algebras.

EXAMPLE 1. Both Theorem 7 and Theorem 12 are false for arbitrary compact convex sets, even in the finite dimension. Take any triangle $K$ in the plane. Let $S$ be the quadrilateral obtained from cutting off a tip of $K$. Then the restriction map $\varphi: A(K) \rightarrow A(S)$ is clearly an isomorphism and one can make $\|\varphi\|\left\|\varphi^{-1}\right\|$ less than 2 by cutting off a small enough tip. However $\partial K$ and $\partial S$ are not homeomorphic.

ExAmple 2. Let $X=\left\{z \in \mathbb{C}: \frac{1}{2} \leq|z| \leq 1\right\}$ and let $A=\{f \in$ $C_{\mathbb{C}}(X): f(z)$ is analytic for $\left.\frac{1}{2}<|z|<1\right\}$. Then the state space $K=\left\{\mu \in A^{*}: \mu(1)=1=\|\mu\|\right\}$ of $A$ is not a simplex, but $\partial K$ is closed and every extreme point of $K$ is a split face [4; p. 108]. 
Let $\mathscr{A}$ be a complex function algebra on a compact Hausdorff space $X$ and let $K=\left\{\mu \in \mathscr{A}^{*}: \mu(1)=1=\|\mu\|\right\}$ be its state space. Then $\partial K$ identifies with the Choquet boundary $\mathrm{Ch} \mathscr{A}$ of $\mathscr{A}$ (cf. $[4 ; \mathrm{p}$. 12]) and the uniform closure $\overline{\mathrm{re} \mathscr{A}}$ of re $\mathscr{A}$ in $C_{\mathbb{R}}(X)$ is isometrically isomorphic to $A(K)$ [4; Theorem I.4.9]. By Bishop's characterization of $\mathrm{Ch} \mathscr{A}$, every $x \in \partial K$ is a weak peak point.

COROllary 13. Let $\mathscr{A}$ and $\mathscr{B}$ be separable function algebras. If there is an isomorphism $\varphi:$ re $\mathscr{A} \rightarrow$ re $\mathscr{B}$ such that $\|\varphi\|\left\|\varphi^{-1}\right\|<2$, then their Choquet boundaries are homeomorphic.

CoRollary 14. Let $\mathscr{A}$ and $\mathscr{B}$ be function algebras with closed Choquet boundaries. If there is an isomorphism $\varphi:$ re $\mathscr{A} \rightarrow \mathrm{re} \mathscr{B}$ with $\|\varphi\|\left\|\varphi^{-1}\right\|<2$, then their Choquet boundaries are homeomorphic.

The above results should be compared with Jarosz's result in [16] that there exists $\varepsilon_{0}>0$ such that if there is a complex isomorphism $\varphi$ between two function algebras $\mathscr{A}$ and $\mathscr{B}$ with $\|\varphi\|\left\|\varphi^{-1}\right\|<1+\varepsilon$, $\varepsilon<\varepsilon_{0}$, then their Choquet boundaries are homeomorphic.

Acknowledgments. We are much indebted to Professor K. S. Lau and Professor C. Lennard for listening to us patiently and helping us with many valuable suggestions. This work was done during the first author's visit to Pittsburgh; he wishes to thank his colleagues for their hospitality and inspiration.

\section{REFERENCES}

[1] E. M. Alfsen, Compact Convex Sets and Boundary Integrals, Springer-Verlag (1971).

[2] D. Amir, On isomorphisms of continuous function spaces, Israel J. Math., 3 (1965), 205-210.

[3] L. Asimow, Decomposable compact convex sets and peak sets for function spaces, Proc. Amer. Math. Soc., 25 (1970), 75-79.

[4] L. Asimow and A. J. Ellis, Convexity Theory and its Applications in Functional Analysis, Academic Press (1980).

[5] E. Behrends, M-structure and the Banach-Stone Theorem, Lecture Notes in Math., vol. 736, Springer-Verlag (1979).

[6] __ Isomorphic Banach-Stone theorem and isomorphisms which are close to isometries, Pacific J. Math., 133 (1988), 229-250.

[7] E. Behrends and M. Cambern, An isomorphic Banach-Stone Theorem, Studia Math., 90 (1988), 15-26.

[8] A. Browder, Introduction to Function Algebras, W. A. Benjamin (1969). 
[9] M. Cambern, On isomorphisms with small bounds, Proc. Amer. Math. Soc., 18 (1967), 1062-1066.

[10] _ Isomorphisms of spaces of continuous vector-valued functions, Illinois $\mathbf{J}$. Math., 20 (1976), 1-11.

[11] H. B. Cohen, $A$ bound-two isomorphism for $C(X)$ Banach spaces, Proc. Amer. Math. Soc., 50 (1975), 215-217.

[12] _ A second-dual method for $C(X)$ isomorphisms, J. Funct. Anal., 23 (1976), 107-118.

[13] L. Drewnowski, A remark on the Amir-Cambern theorem, Funct. Approx. Comment. Math., 16 (1988), 181-190, Adam Mickiewicz University Press.

[14] P. Harmond and A. Lima, Banach spaces which are M-ideals in their biduals, Trans. Amer. Math. Soc., 283 (1984), 253-264.

[15] K. Jarosz, A generalization of the Banach-Stone theorem, Studia Math., 73 (1982), 33-39.

[16] _ Perturbations of Banach Algebras, Lecture Notes in Math., vol. 1120, (1985), Springer-Verlag.

[17] _ Small isomorphisms of $C(X, E)$ spaces, Pacific J. Math., 138 (1989), 295-315.

Received December 27, 1990 and in revised form September 3, 1991.

Goldsmiths' College

UNIVERSITY OF LONDON

LONDON SE14, ENGLAND

AND

UNIVERSITY OF PitTSBURgh

Pittsburgh, PA 15260 



\section{PACIFIC JOURNAL OF MATHEMATICS EDITORS}

V. S. VARADARAJAN (Managing Editor)

University of California

Los Angeles, CA 90024-1555

vsv@math.ucla.edu

Herbert Clemens

University of Utah

Salt Lake City, UT 84112

clemens@math.utah.edu

F. Michael Christ

University of California

Los Angeles, CA 90024-1555

christ@math.ucla.edu

THOMAS ENRIGHT

University of California, San Diego

La Jolla, CA 92093

tenright@ucsd.edu
Nicholas ERcolani

University of Arizona

Tucson, AZ 85721

ercolani@math.arizona.edu

R. FINN

Stanford University

Stanford, CA 94305

finn@gauss.stanford.edu

VAUGHAN F. R. JoNES

University of California

Berkeley, CA 94720

vfr@math.berkeley.edu

STEVEN KeRCKHOFF

Stanford University

Stanford, CA 94305

spk@gauss.stanford.edu
C. C. MOORE

University of California

Berkeley, CA 94720

Martin ScharlemanN

University of California

Santa Barbara, CA 93106

mgscharl@henri.ucsb.edu

Harold Stark

University of California, San Diego

La Jolla, CA 92093

\begin{tabular}{|c|c|c|c|c|}
\hline \multicolumn{5}{|c|}{ ASSOCIATE EDITORS } \\
\hline R. ARENS & $\begin{array}{l}\text { E. F. BECKENBACH } \\
(1906-1982)\end{array}$ & B. H. NeumanN & $\begin{array}{c}\text { F. WoLF } \\
(1904-1989)\end{array}$ & K. YoshidA \\
\hline \multicolumn{5}{|c|}{ SUPPORTING INSTITUTIONS } \\
\hline \multicolumn{2}{|c|}{ UNIVERSITY OF ARIZONA } & \multicolumn{3}{|c|}{ UNIVERSITY OF OREGON } \\
\hline \multicolumn{2}{|c|}{ UNIVERSITY OF BRITISH COLUMB } & \multicolumn{3}{|c|}{$\begin{array}{l}\text { UNIVERSITY OF SOUTHERN CALIFORNI } \\
\text { STANFORD UNIVERSITY }\end{array}$} \\
\hline \multicolumn{2}{|c|}{ CALIFORNIA INSTITUTE OF TECHNOLOGY } & \multirow{2}{*}{\multicolumn{3}{|c|}{$\begin{array}{l}\text { STANFORD UNIVERSITY } \\
\text { UNIVERSITY OF HAWAII }\end{array}$}} \\
\hline \multicolumn{2}{|c|}{ UNIVERSITY OF CALIFORNIA } & & & \\
\hline \multicolumn{2}{|c|}{ MONTANA STATE UNIVERSITY } & \multicolumn{3}{|c|}{ UNIVERSITY OF TOKYO } \\
\hline \multicolumn{2}{|c|}{ UNIVERSITY OF NEVADA, RENO } & \multicolumn{3}{|c|}{ UNIVERSITY OF UTAH } \\
\hline \multicolumn{2}{|c|}{ NEW MEXICO STATE UNIVERSITY } & \multicolumn{3}{|c|}{ WASHINGTON STATE UNIVERSITY } \\
\hline \multicolumn{2}{|c|}{ OREGON STATE UNIVERSITY } & \multicolumn{3}{|c|}{ UNIVERSITY OF WASHINGTON } \\
\hline
\end{tabular}




\section{PACIFIC JOURNAL OF MATHEMATICS}

Volume $155 \quad$ No. $1 \quad$ September 1992

Characterization of modular correspondences by geometric properties 1 Allan Russell AdLer

Representations of convex nondentable sets

SPIROS ARGYROS and IRENE DELIYANNI

Isomorphisms of spaces of continuous affine functions

CHO-Ho CHU and HENRY BRUCE COHEN

Universal classes of Orlicz function spaces

FRANCISCO LUIS HERNÁNDEZ RODRÍGUEZ and CESAR RUIZ

Asymptotic behavior of the curvature of the Bergman metric of the thin 99 domains

KANG-TAE KIM

Quadratic central polynomials with derivation and involution

CHARLES PHILIP LANSKI

Nonsplit ring spectra and products of $\beta$-elements in the stable homotopy of Moore spaces

\section{JIN KUN LIN}

Orientation and string structures on loop space

DENNIS MCLAUGHLIN

Homomorphisms of Bunce-Deddens algebras

\section{CORNEL PASNICU}

Certain $C^{*}$-algebras with real rank zero and their corona and multiplier 169 algebras. Part I

SHUANG ZHANG

Correction to: "On the density of twistor elementary states"

Michael G. Eastwood and A. M. Pilato 